\title{
Impact of gastric resection and enteric anastomotic configuration on delayed gastric emptying after pancreaticoduodenectomy: a network meta-analysis of randomized trials
}

\author{
C. Varghese $\mathbb{D}^{1}$, S. Bhat $\mathbb{D D}^{1}$, T. H.-H. Wang ${ }^{1}$, G. O'Grady ${ }^{1}$ and S. Pandanaboyana ${ }^{2,3, *}$ \\ ${ }^{1}$ Department of Surgery, Faculty of Medical and Health Sciences, University of Auckland, Auckland, New Zealand \\ ${ }^{2} \mathrm{HPB}$ and Transplant Unit, Freeman Hospital, Newcastle upon Tyne, UK \\ ${ }^{3}$ Population Health Sciences Institute, Newcastle University, Newcastle upon Tyne, UK \\ *Correspondence to: HPB and Transplant Unit, Freeman Hospital, Newcastle upon Tyne NE7 7DN, UK (e-mail: sanjay.pandanaboyana@ncl.ac.uk)
}

\begin{abstract}
Introduction: Delayed gastric emptying (DGE) is frequent after pancreaticoduodenectomy (PD). Several RCTs have explored operative strategies to minimize DGE, however, the optimal combination of gastric resection approach, anastomotic route, configuration and the use of enteroenterostomy remains unclear.

Methods: MEDLINE, Embase and CENTRAL databases were systematically searched for RCTs comparing gastric resection (classic Whipple, pylorus-resecting, pylorus-preserving), anastomotic route (antecolic, retrocolic), configuration (loop gastroenterostomy/ Billroth II, Roux-en-Y), and use of enteroenterostomy (Braun). A random-effects, Bayesian network meta-analysis with non-informative priors was conducted to determine the optimal combination of approaches to PD for minimizing DGE.

Results: Twenty-four RCTs, including 2526 patients and 14 approaches were included. There was some heterogeneity, although inconsistency was low. The overall incidence of DGE was 25.6 per cent (647 patients). Pylorus-resecting, antecolic, Billroth II with Braun enteroenterostomy was associated with the lowest rates of DGE and ranked the best in 35 per cent of comparisons. Classic Whipple, retrocolic, Billroth II with Braun ranked the worst for DGE in 32 per cent of comparisons. Pairwise meta-analysis of retrocolic versus antecolic route for gastrojejunostomy found increased risk of DGE with the retrocolic route (odds ratio 2.10, 95 per cent credibility interval (cr.i.) 0.92 to 4.70). Pairwise meta-analysis of enteroenterostomy found a trend towards lower DGE rates when this was used (odds ratio 1.90, 95 per cent cr.i. 0.92 to 3.90). Having a Braun enteroenterostomy ranked the best in 96 per cent of comparisons.
\end{abstract}

Conclusion: Based on existing RCT evidence, a pylorus-resecting, antecolic, Billroth II with Braun enteroenterostomy seems to be associated with the lowest rates of DGE.

Preregistration: PROSPERO submitted 23 December 2020. CRD42021227637

\section{Introduction}

Pancreaticoduodenectomy (PD) is commonly performed for benign and malignant pancreatic head and periampullary disease. There remains a high prevalence of postoperative delayed gastric emptying (DGE), affecting between 10 and 45 per cent of patients $^{1-5}$. DGE is associated with poorer quality of life $^{6}$, increased hospital length of stay (LOS) ${ }^{7}$, readmissions ${ }^{8}$ and healthcare $\operatorname{costs}^{9,10}$. Technical approaches to gastric resection and reconstruction in $\mathrm{PD}$ are thought to impact the rates of $\mathrm{DGE}^{11}$.

The recent pylorus resection or pylorus preservation (PROPP) RCT and several meta-analyses have shown comparable DGE rates between pylorus-preserving and pylorus-resecting operations, although comparisons between the different resections are limited ${ }^{3,12,13}$. Previous meta-analyses have also suggested that antecolic gastrojejunostomy is the more effective route for minimizing DGE ${ }^{14-16}$, but again evidence is conflicting ${ }^{17}$. Similarly, it remains controversial whether a simple Billroth II or Roux-en-Y reconstruction makes a difference ${ }^{16,18-21}$ and what value is gained from Braun enteroenterostomy, to prevent bile reflux, in terms of DGE rates ${ }^{22}$

It remains unclear which combination of gastric resection and method of reconstruction is optimal for reducing DGE after PD. Traditional meta-analyses are limited to pairwise comparisons and large-scale surgical RCTs of all potential combinations are impractical. Network meta-analyses (NMAs) allow comparison and ranking of a range of surgical approaches simultaneously, through direct and indirect comparisons. This NMA therefore aimed to identify the optimal combination of gastric resection, route of anastomosis, anastomotic configuration and use of Braun enteroenterostomy on DGE after PD. Other specific complications of PD were also examined to see how they were influenced by the resection and reconstruction methods that affected rates of DGE. 


\section{Methods}

This network meta-analysis followed PRISMA guidelines ${ }^{23,24}$. The study protocol was prospectively registered on PROSPERO (ID: CRD42021227637) before database searching.

\section{Literature search}

A systematic search of MEDLINE (OVID), EMBASE (1980-2020), EMBASE Classic (1947-1973), and the Cochrane Controlled Register of Trials (CENTRAL) was conducted from their date of inception to December 2020. The following query terms were employed: the combined results of 'pancreaticoduodenectomy' OR 'Whipple' OR 'gastroenteric' OR 'antecolic' OR 'retrocolic' OR 'Billroth' OR 'Roux-en-Y' OR 'pylorus-preserving' or 'antrectomy' AND the combined results of 'gastroparesis' OR 'delayed gastric emptying' or 'DGE' AND the combined results of 'trials' OR 'randomised' OR 'randomized controlled trial'. 'Explode' functions and Medical Subject Heading (MeSH) terms were used where available. There were no date or language restrictions. Article and review reference lists were also screened by two authors independently to identify additional potentially relevant materials (Supporting information S1).

\section{Inclusion and exclusion criteria}

RCTs that described the gastric resection technique, route of gastrojejunostomy (antecolic, retrocolic), the technique of reconstruction (loop gastroenterostomy/Billroth II, Roux-en-Y), and addition of Braun enteroenterostomy in adults (over 16 years old) undergoing PD were considered for inclusion. Only RCTs where DGE was either the primary endpoint or the study was adequately powered to detect a 20 per cent difference in the rates of DGE between groups at 80 per cent power and an alpha of 0.05 were eligible for inclusion (accepting a typical DGE incidence of around 30 per cent ${ }^{3}$ ). If aspects of the operative approach being investigated were unclear, individual authors were contacted for further details.

Studies were excluded if they were non-randomized, included paediatric populations (less than 16 years), were animal studies, or included operations other than PD. Only trials comparing surgical techniques were included. Trials comparing pharmacological interventions or methods of pancreatic anastomosis were excluded. Trials where the surgeon could choose which method of gastric resection, route or technique of anastomotic reconstruction were excluded. Studies reporting duplicate outcomes from a previously published report were also excluded, to minimize publication bias.

\section{Study selection and data extraction}

Two authors screened titles and abstracts and reviewed full texts identified in the literature search for inclusion and extracted data onto a prespecified template independently. Discrepancies were discussed and resolved by mediation with a third independent reviewer. If there were missing data, the authors of the articles were contacted. Primary efficacy outcome data on rates of overall DGE were extracted, as well as clinically relevant DGE (grade $\mathrm{B} / \mathrm{C}$ as defined by the International Study Group of Pancreatic Surgery (ISGPS) ${ }^{25}$ ). Secondary outcomes included overall rates of postoperative pancreatic fistula (POPF) as well as clinically relevant POPF (grade $\mathrm{B} / \mathrm{C}$ as defined by the International Study Group of Pancreatic Fistula (ISGPF ${ }^{26}$ ), duration of operation, intraoperative blood loss, intra-abdominal abscess, wound infection, haemorrhage ${ }^{27}$, hospital LOS, reoperation and mortality. Any definition of mortality provided by authors, for any follow-up duration, was included. The Cochrane Collaboration's Risk-of-bias tool 2.0 was used to assess study design ${ }^{28}$.

\section{Terminology and definitions}

Gastric resection was defined as classic Whipple (CW), pylorusresecting (PR), and pylorus-preserving (PP). CW included studies with antrectomy or 20-40 per cent distal gastrectomy. PR (synonymous with subtotal stomach-preserving PD) included studies where gastric resection occurred less than $3 \mathrm{~cm}$ proximal to the pyloric ring. PP PD studies were defined based on gastric resection occurring at any distance distal to the pyloric ring. Route of anastomosis was defined as antecolic or retrocolic if the route of the gastrojejunostomy was anterior or posterior to the transverse colon, respectively. Reconstructions were defined as Billroth II if the pancreaticojejunostomy and hepaticojejunostomy were proximal to an end-to-side gastrojejunostomy in the absence of separate Roux and afferent limbs. The term Roux-en-Y reconstruction was used if a Roux loop was used to separate pancreatic and hepatic outflows. Braun enteroenterostomy was defined as a jejuno-jejunostomy distal to the gastroenteric anastomosis.

\section{Statistical analysis}

A random-effects NMA was performed using GeMTC in R ( $R$ Foundation for Statistical Computing, Vienna, Austria) ${ }^{29}$. GeMTC employs a Just Another Gibbs Sampler software to conduct armbased calculations using a Bayesian framework and non-informative priors. Where studies reported medians, mean estimates were derived from the methods of Wan et al. and Luo et al. ${ }^{30,31}$. Network maps were generated to visualize all direct comparisons made. Line thickness corresponded with the number of studies assessing a particular direct comparison and the size of nodes correlated with the number of participants receiving a particular intervention. A continuity correction of one was applied where a categorical outcome was achieved by none of the participants in a trial $\mathrm{arm}^{32}$. Odds ratios (OR) were used for categorical outcome data, and mean differences (MD) for continuous data, both accompanied by 95 per cent credibility intervals (cr.i.). CW with antecolic gastrojejunostomy and Billroth II reconstruction without Braun enteroenterostomy was considered the comparator arm where applicable. Rankogram plots visualized the relative effectiveness of each intervention per outcome represented as stacked bar plots of the probability of each intervention achieving each rank. Sum under the cumulative ranking scores were used to rank interventions where a score of 1 meant the intervention was the best ranked 100 per cent of the time, and a score of 0 where it ranked as the worst intervention 100 per cent of the time $^{33}$. Heterogeneity was assessed via the random-effects standard deviation as described by Valkenhoef and colleagues ${ }^{34}$. A node-splitting analysis of inconsistency was used to assess the comparability of indirect and direct comparisons. Transitivity was assessed by collecting and comparing demographic data, surgical approach and co-interventions across direct comparisons. Comparison-adjusted funnel plots were constructed and visually inspected for asymmetry to indicate publication bias. A sensitivity analysis was performed by sequential removal of single studies to review the resulting discrepancies in rankings. A subgroup analysis and regression excluding studies that compared Braun versus no Braun enteroenterostomy was also performed. If 95 per cent credibility intervals did not cross the no-effect line ( 0 for continuous outcomes and 1 for dichotomous outcomes), results were considered statistically significant. 


\section{Results}

Search results and study selection are summarized in Fig. 1. The search identified 1215 studies, of which 24 RCTs met the inclusion criteria ${ }^{2-5,35-54}$. A total of 2647 patients were randomized and 2526 included in the analysis. These RCTs were published between 1999 and 2020 and compared 14 different combinations of gastric resection and anastomotic configurations after PD.

Direct comparisons from included RCTs are summarized in Table 1. The most common direct comparison was between PP PD with antecolic Billroth II reconstruction and PP PD with retrocolic Billroth II (both without Braun enteroenterostomy) in four studies.

\section{Transitivity analysis}

Definitions for the primary outcome of overall DGE were largely similar, although only 14 studies used the ISGPS definition. Similarly, 15 studies used the ISGPF definition for POPF. Only five studies reported the rates of prokinetic use after $\mathrm{PD}^{2-5,38}$ and three reported rates of parenteral nutrition (30-45 per cent), with no differences between comparator arms ${ }^{3,43,47}$. Ages, gender mix and BMI were generally comparable between direct comparisons, although the single study comparing PR, retrocolic, Billroth II against PR, antecolic Billroth II had a male-predominant cohort (more than 70 per cent). Most studies were conducted in Asia (16 of 24; 67 per cent) $5,35,36,38-46,48,50,51,53,10$ of which were in

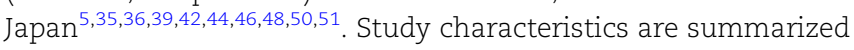
in Table S1.

\section{Risk of bias}

Risk-of-bias assessments are shown in Figs S1 and S2. Four studies (17 per cent) were considered to be at low risk of bias, with the remaining 20 (83 per cent) considered to be at high risk of bias, predominantly due to the lack of blinding. Most studies demonstrated clear and efficacious randomization, appropriate intention-to-treat analysis and transparent outcome reporting.

\section{Primary outcome measure Overall delayed gastric emptying}

Twenty-four trials comparing 14 combinations of PD in 2526 patients reported overall rates of DGE. The overall incidence of DGE was 25.6 per cent (647 of 2526 patients). The overall incidence of clinically relevant DGE was 14.9 per cent (253 of 1698). Table 2 summarizes the results of the NMA from the direct comparisons of overall DGE rates. PP, antecolic, Billroth II versus PP, retrocolic, Billroth II had the most direct comparisons (Fig. 2). Rankograms showed that PR, antecolic, Billroth II with Braun enteroenterostomy was associated with the lowest rates of overall DGE (Fig. 3), followed by CW, antecolic, Billroth II with Braun enteroenterostomy. PR, antecolic, Billroth II with Braun enteroenterostomy ranked the best approach in 35 per cent of comparisons. CW, retrocolic, Billroth II with Braun ranked the worst approach for overall DGE in 32 per cent of comparisons (Supporting information S2).

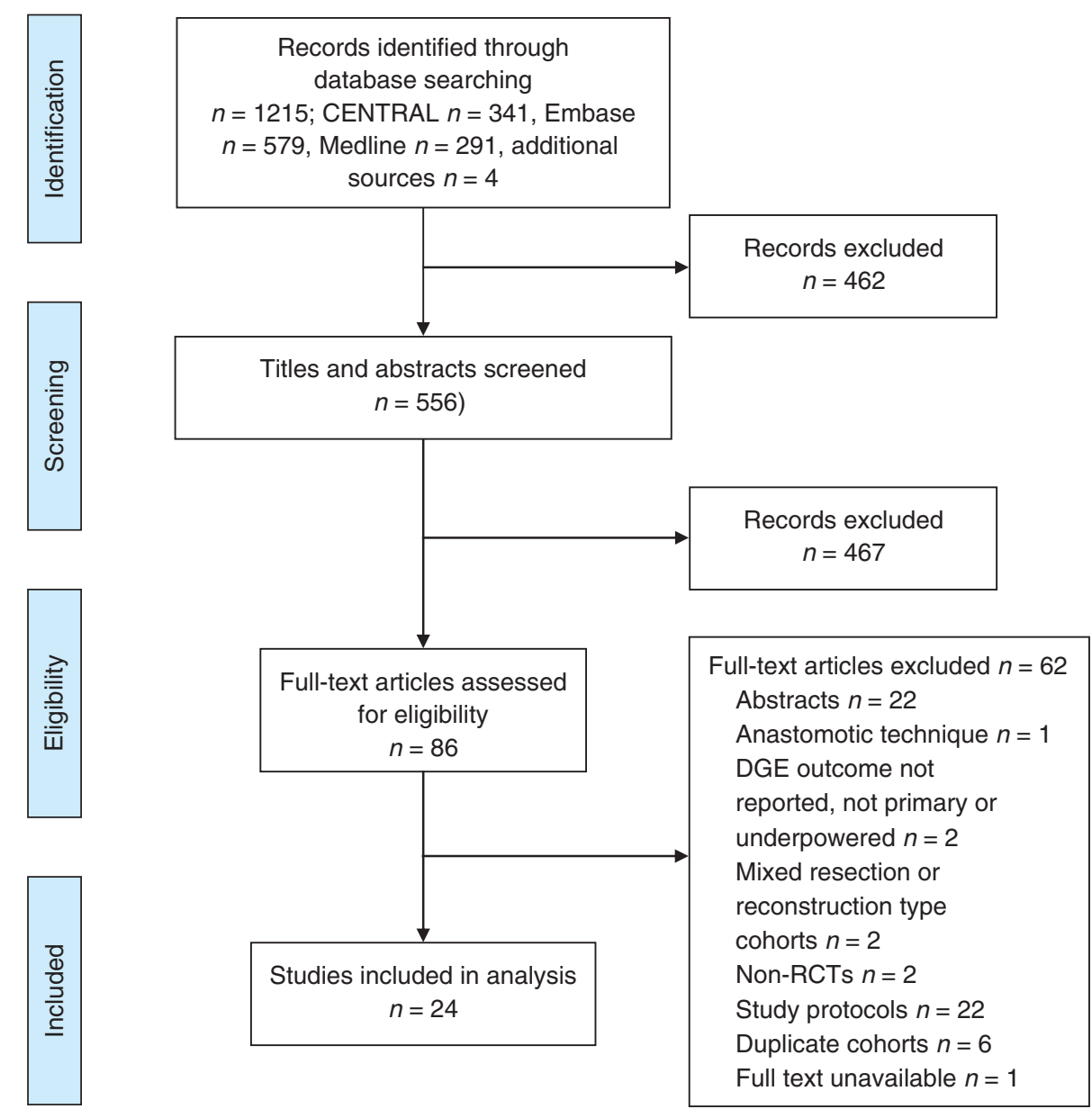

Fig. 1 PRISMA flow diagram of study selection 
Table 1 Summary of included trials

\begin{tabular}{|c|c|c|c|c|c|c|c|c|c|}
\hline \multirow[b]{2}{*}{ Trial comparison } & \multirow[b]{2}{*}{$\begin{array}{l}\text { No. of } \\
\text { trials }\end{array}$} & \multirow[b]{2}{*}{$\begin{array}{c}\text { No. of } \\
\text { patients* }\end{array}$} & \multirow[b]{2}{*}{$\begin{array}{c}\text { Publication } \\
\text { years }\end{array}$} & \multirow[b]{2}{*}{ Age (years) ${ }^{\dagger}$} & \multirow[b]{2}{*}{ Women (\%) } & \multirow[b]{2}{*}{ BMI $\left(\mathrm{kg} / \mathrm{m}^{2}\right)^{\dagger}$} & \multicolumn{3}{|c|}{ Trial location } \\
\hline & & & & & & & Asia & $\begin{array}{c}\text { North } \\
\text { America }\end{array}$ & Europe \\
\hline PP_A_BII versus PP_R_BII & 4 & 393 & 2006-2019 & 62.1 & 42.1 & 25 & 2 & & 2 \\
\hline CW_A_BII versus CW_A_RY & 3 & 360 & 2013-2018 & 66.9 & 64.2 & 24.7 & 1 & & 2 \\
\hline CW_A_BII versus PP_A_BII & 2 & 201 & 1999-2004 & 59.7 & 37.3 & & 1 & & 1 \\
\hline PP_A_BII versus PR_A_BII & 2 & 328 & 2011-2018 & 65.4 & 45 & 25.5 & 1 & & 1 \\
\hline PP_A_BII_B versus PP_R_BII_B & 2 & 155 & 2009-2013 & 67.4 & 41.7 & 21.9 & 2 & & \\
\hline CW_A_BII_B versus CW_A_BII & 1 & 30 & 2015 & 56.3 & 33.3 & NA & 1 & & \\
\hline CW_A_RY versus CW_A_RY_B & 1 & 104 & 2017 & 53.9 & 38.5 & NA & 1 & & \\
\hline CW_R_BII versus CW_A_BII & 1 & 214 & 2020 & NA & 38.2 & 21.8 & 1 & & \\
\hline CW_R_BII_B versus PP_R_BII_B & 1 & 214 & 2005 & 61 & 46.9 & NA & & & 1 \\
\hline PP_A_BII_B versus PP_A_BII & 1 & 60 & 2016 & 66 & 36.7 & NA & 1 & & \\
\hline PP_R_BII versus CW_R_BII & 1 & 114 & 1999 & 65 & 46.5 & NA & & 1 & \\
\hline PP_R_BII versus PR_R_BII & 1 & 106 & 2014 & 66.5 & 36 & 22.8 & 1 & & \\
\hline PR_A_BII versus PR_A_BII_B & 1 & 68 & 2017 & 67.1 & 35.3 & 21.7 & 1 & & \\
\hline PR_A_BII versus PR_A_RY & 1 & 153 & 2014 & 68.8 & 46.4 & NA & 1 & & \\
\hline PR_A_BII_B versus PR_A_RY & 1 & 101 & 2013 & 66.1 & 40.6 & 21.5 & 1 & & \\
\hline PR_R_BII versus PR_A_BII & 1 & 46 & 2011 & NA & 29.2 & 23.1 & 1 & & \\
\hline
\end{tabular}

Number of patients randomized and used in intention-to-treat analysis. ${ }^{\dagger}$ Values are means. PP, pylorus preserving; CW, classic Whipple; PR, pylorus resecting; A, antecolic; R, retrocolic; BII, Billroth II; RY, Roux-en-Y; B, Braun enteroenterostomy.

\section{Secondary outcome measures Overall postoperative pancreatic fistula}

Overall POPF rates were reported in 22 trials comparing 13 approaches amongst 2472 patients. The overall incidence of POPF was 23.2 per cent (571 of 2466 patients). PP, retrocolic Billroth II versus PP antecolic Billroth II, both without Braun was the most frequent direct comparison. Rankograms showed that CW, retrocolic, Billroth II with Braun enteroenterostomy was associated with the lowest rates of POPF (in 79 per cent of comparisons); this was followed by PR, antecolic, Roux-en-Y without Braun. CW, retrocolic, Billroth II with Braun was only reported in a single study ${ }^{47}$ and a sensitivity analysis excluding this study showed that PP, retrocolic, Billroth II with Braun was associated with the lowest POPF rates in 51 per cent of comparisons, followed again by PR, antecolic, Roux-en-Y without Braun in 26 per cent of comparisons. CW, antecolic, Roux-en-Y with Braun ranked the worst approach in 28 per cent of comparisons and persisted as the worst ranked approach following sensitivity analysis (25 per cent of comparisons) (Supporting information S2).

\section{Intra-abdominal abscess}

Eighteen trials reported intra-abdominal abscess rates with direct comparisons of 11 approaches comprising 2105 patients. The overall incidence of intra-abdominal abscesses was 13.6 per cent (285 of 2103 patients). Billroth II versus Roux-en-Y configuration of CW with antecolic gastrojejunostomy and without Braun was the most frequent direct comparison. PR, antecolic, Roux-en-Y without Braun ranked best in 33 per cent of direct comparisons followed by PP, retrocolic, Billroth II with Braun in 30 per cent of direct comparisons. Because PR, antecolic, Roux-en-Y without Braun was only reported in a single study ${ }^{50}$, a sensitivity analysis with this study removed resulted in PP, retrocolic, Billroth II with Braun ranking first in 33 per cent of comparisons. PR, retrocolic, Billroth II without Braun was consistently associated with higher rates of intra-abdominal abscesses (63 per cent of direct comparisons in both the overall and sensitivity analysis) and had significantly higher rates compared with PR, antecolic, Roux-en-Y without Braun (OR 9.32, 95 per cent cr.i. 1.08 to 136.52) and PR, antecolic, Billroth II without Braun (OR 6.01, 95 per cent cr.i. 1.06 to 65.83 )

\section{Post-pancreatectomy haemorrhage}

Direct comparisons of post-pancreatectomy haemorrhage (PPH) rates were reported in 15 trials, comparing 11 approaches in 1689 patients. The overall incidence of PPH was 6.2 per cent (105 of 1683 patients). Rankograms showed comparable PPH rates with PP, retrocolic, Billroth II with Braun (ranked best in 29 per cent of comparisons) and CW, antecolic, Billroth II with Braun (ranked best in 27 per cent of comparisons). PR, antecolic Roux-en-Y ranked worst for $\mathrm{PPH}$ in 61 per cent of comparisons; however, it was only reported in one study. On sensitivity analysis, PP, retrocolic, Billroth II without Braun ranked worst in 27.6 per cent of comparisons.

\section{Mortality}

All 24 trials reported mortality, with direct comparisons of all 14 approaches, including 2526 patients. The overall incidence of mortality (reported at any time up to 90 days) was 2.1 per cent (54 of 2526 patients). Antecolic versus retrocolic, PP, Billroth II without Braun was the most frequent direct comparison. CW, antecolic, Billroth II with Braun had the lowest mortality rate in 27 per cent of direct comparisons and CW, retrocolic, Billroth II with Braun had the worst mortality rate in 27 per cent of direct comparisons.

\section{Length of stay}

Of 20 trials that reported LOS (days), 12 made direct comparisons of specific approaches, involving 2331 patients. Billroth II versus Roux-en-Y configuration for CW, antecolic, without Braun was the most frequent direct comparison. CW, retrocolic, Billroth II with Braun had the shortest LOS in 63 per cent of all comparisons, followed by PP, retrocolic, Billroth II with Braun in 25 per cent of all comparisons. PR, antecolic, Roux-en-Y was associated with the longest LOS in 36 per cent of all comparisons. PR, antecolic, Roux-en-Y was associated with significantly longer LOS compared with the best-ranked approach: CW, retrocolic, Billroth II with Braun (MD 13.14 days, 95 per cent cr.i. 0.12 to 27.16).

Results of further secondary outcomes including duration of operation, blood loss, bile leak, wound infection and reoperation rates can be found in Supporting information S3 and Table 3. 


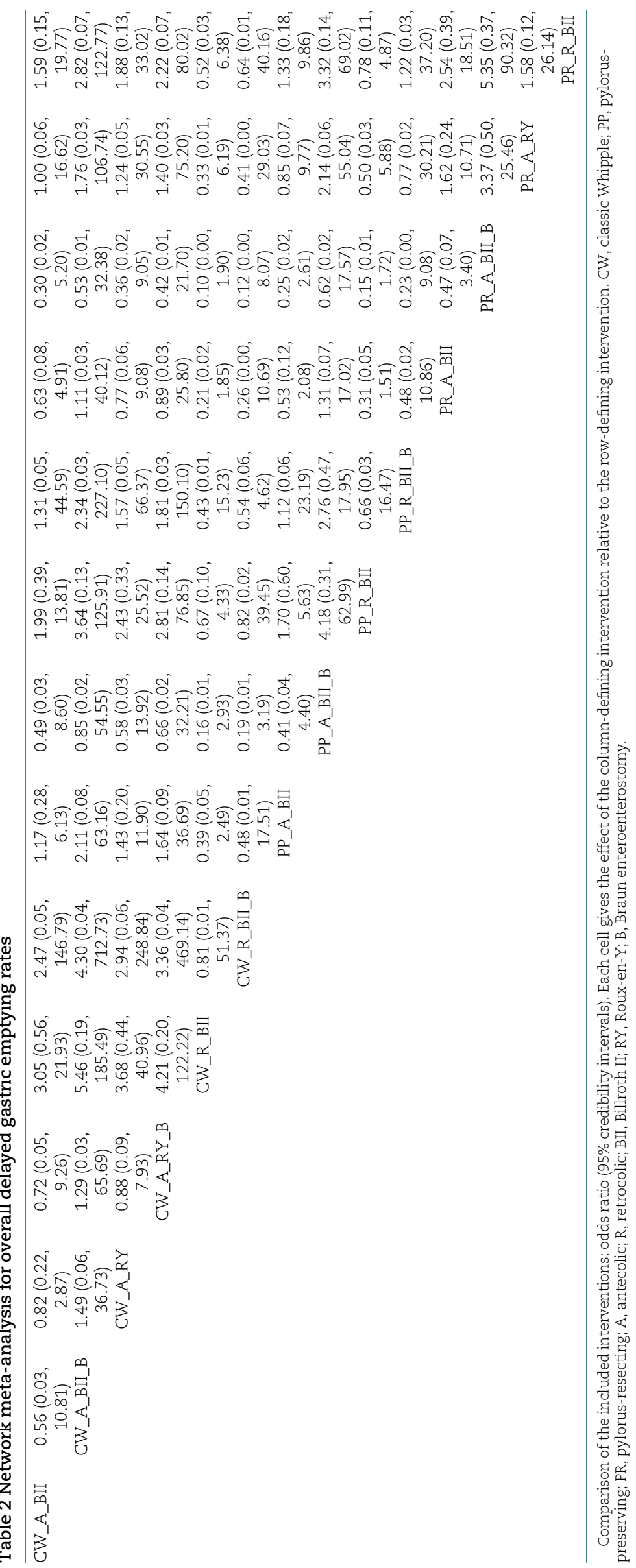




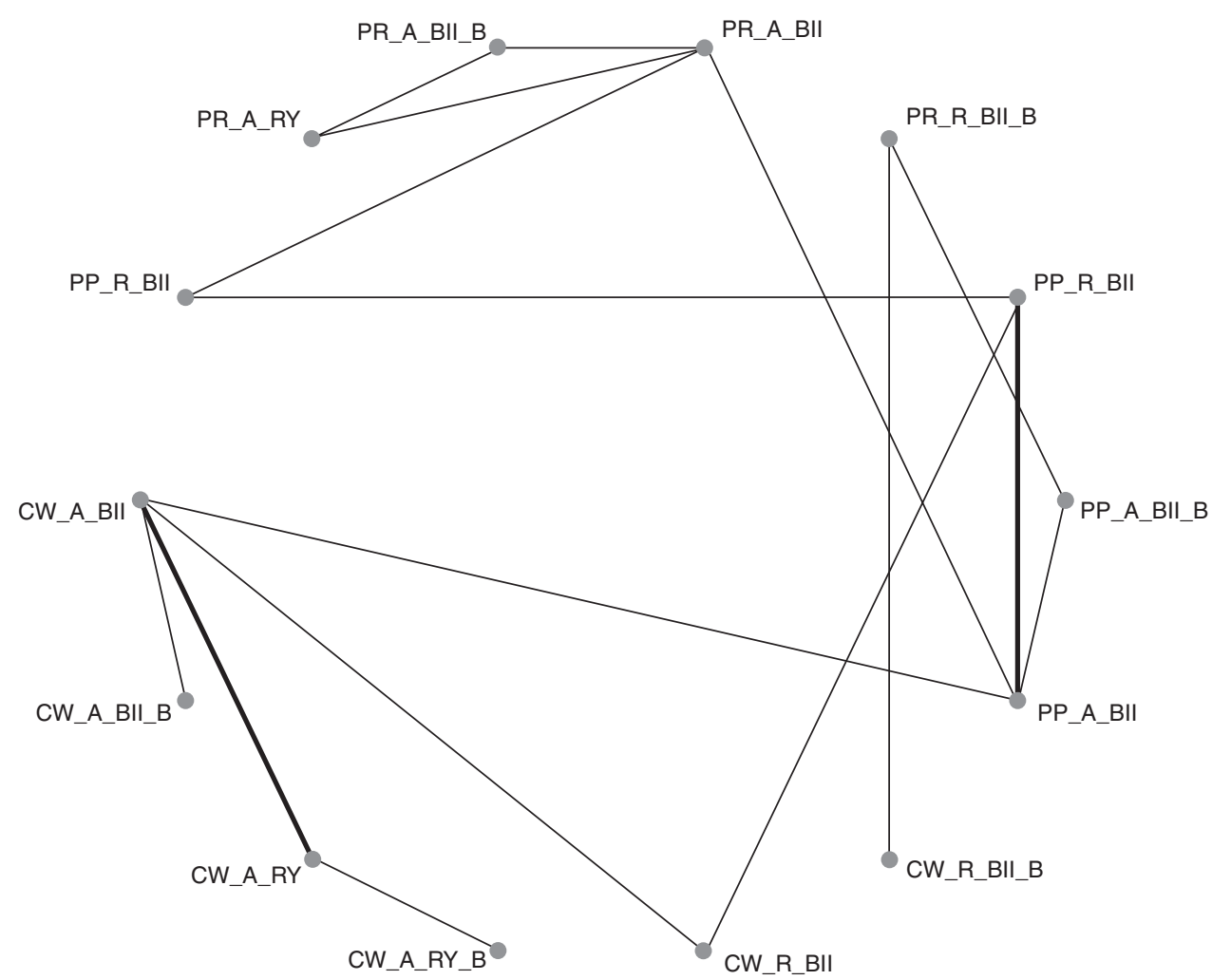

Fig. 2 Network map for overall delayed gastric emptying

PP, pylorus preserving; CW, classic Whipple; PR, pylorus resecting; A, antecolic; R, retrocolic; BII, Billroth II; RY, Roux-en-Y; B, Braun enteroenterostomy Note: Figure Replacement Requested.

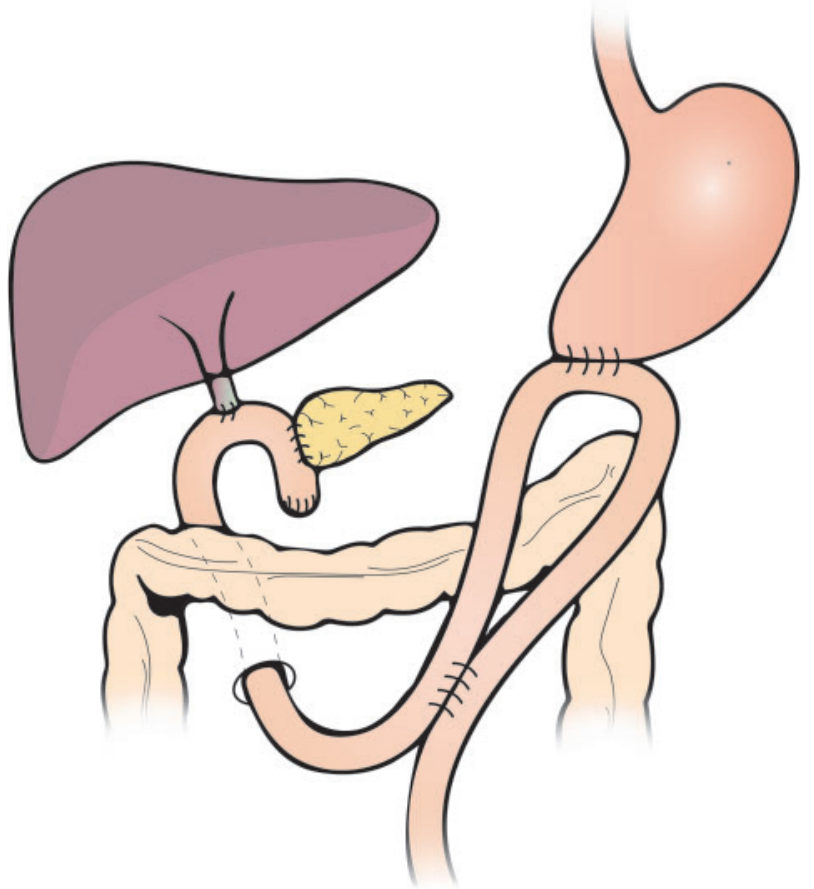

Fig. 3 Schematic diagram of pylorus-resecting, antecolic, Billroth II anastomosis with Braun enteroenterostomy

\section{Subgroup analyses Impact of Braun enteroenterostomy on DGE}

Pairwise meta-analysis of Braun enteroenterostomy found slightly lower DGE rates with Braun compared with no Braun (OR 1.90, 95 per cent cr.i. 0.92 to 3.90). Having a Braun enteroenterostomy ranked the best approach in reducing DGE in 96 per cent of comparisons. Results of a subgroup analysis excluding Braun comparisons showed PR, antecolic, Billroth II to rank the best in reducing DGE (Supporting information S2).

\section{Impact of pyloric resection on DGE}

In studies that directly compared CW, PR and PP approaches with gastric resection in $\mathrm{PD}$, no statistically significant differences in the rates of overall DGE were found. However, PR PD ranked as the best approach for reducing DGE in 71 per cent of comparisons.

Impact of antecolic versus retrocolic gastrojejunostomy on DGE

Pairwise meta-analysis of antecolic versus retrocolic route of gastrojejunostomy found increased risk of DGE with the retrocolic compared with the antecolic route (OR 2.10, 95 per cent cr.i. 0.92 to 4.70). While this did not reach statistical significance, antecolic anastomosis ranked the best route for reducing DGE in 98 per cent of comparisons.

\section{Impact of Billroth II versus Roux-en-Y reconstruction on DGE}

Pairwise meta-analysis of Billroth II versus Roux-en-Y anastomotic configuration found no discernible differences in the rates of DGE (OR 1.2, 95 per cent cr.i. 0.59 to 2.30). Billroth II ranked the best configuration for reducing DGE in 68 per cent of comparisons.

These results are congruent with PR, antecolic, Billroth II with Braun enteroenterostomy being the optimal combination of $\mathrm{PD}$ 
approaches to minimize DGE, demonstrating consistency with the NMA.

\section{Risk of heterogeneity, inconsistency and publication bias}

Some heterogeneity was found for the outcomes: rates of overall DGE, PPH, intraoperative blood loss, wound infection, bile leak, and in the separated analysis assessing gastric resection comparisons only. No significant heterogeneity was identified in the other outcome data. Node-splitting analysis models demonstrated no inconsistency in most outcomes except LOS. This outcome should therefore be interpreted with caution. Heterogeneity and inconsistency results are summarized in Supporting information S3. Comparison-adjusted funnel plots showed an even distribution of studies adjacent to the pooled estimate line for most outcomes (Supporting information S2), although some funnel plot asymmetry was evident in trials comparing antecolic versus retrocolic CW, Billroth II without Braun and antecolic versus retrocolic PP, Billroth II without Braun for the primary outcome of overall DGE incidence.

\section{Discussion}

This NMA compared the impact of gastric resection, route and configuration of enteric anastomosis, and the addition of Braun enteroenterostomy on DGE following PD and found that DGE Occurred in nearly a quarter of patients. PR, antecolic, Billroth II with Braun enteroenterostomy ranked best for minimizing DGE, in both the overall analysis and subgroup analyses of techniques comparing each aspect of PD independently. PP, retrocolic, Billroth II with Braun enteroenterostomy ranked best on sensitivity analysis for minimizing complication rates such as POPF, intra-abdominal abscesses, bile leak, PPH, duration of operation and intraoperative blood loss. Postoperative complications such as POPF and intra-abdominal abscesses are known risk factors for $D G E^{55}$, and optimal approaches to PD should ideally minimize all of these complications. CW, retrocolic, Billroth II with Braun ranked worst for DGE and mortality, while PR, retrocolic, Billroth II without Braun ranked worst for intra-abdominal abscess, bile leak, intraoperative blood loss and reoperation.

Other studies have examined approaches that might influence the risk of DGE. Some meta-analyses have suggested antecolic, Billroth II to be favourable for DGE ${ }^{14-16}$. Of meta-analyses that specifically investigated resection of the pylorus, Klaiber and colleagues found no difference between PR and PP in a subgroup analysis of RCTs ${ }^{13}$, similar to the findings of this present network meta-analysis. When pooled with non-randomized studies, both Klaiber and colleagues and Huang and colleagues found PR PD to be preferable in minimizing DGE ${ }^{12,13}$. A further study compared PR, CW and PP PD in a series of subgroup analyses and found PR, but not CW, to improve DGE compared with $\mathrm{PP}^{56}$. These previous studies are confounded by non-RCT data with inherent selection bias. Where more than two operative options exist at each stage of $\mathrm{PD}$, pairwise meta-analysis is unable to provide a suitable answer. The present study employed a Bayesian network metaanalysis of RCTs to compare 14 different approaches to gastric resection and enteric anastomotic route and configuration in PD. This methodology is advantageous as it allows simultaneous comparison of all randomized data available on this topic, while maintaining randomization during pooled analysis ${ }^{57}$. Direct and indirect comparisons from the RCTs facilitated accurate network meta-analysis as evidenced by the lack of inconsistency in all outcomes except LOS. Despite these advantages, studies included 
in the present analysis were still deemed to be at high risk of bias, predominantly due to limited blinding of surgeons and investigators collecting outcome data. While the majority of RCTs employed randomization intraoperatively prior to the step under investigation, there remains a risk of selection and detection bias due to the limited blinding. Otherwise, there were minimal causes of concern as most studies conducted intention-to-treat analysis, demonstrated adequate and transparent randomization and patient blinding, and reported outcomes in-full, with few missing data. Indications for $\mathrm{PD}$, aspects of postoperative care such as use of prokinetics, complication grades and rates of postoperative enteral nutrition were variably and poorly reported. These factors may be modifiers of treatment effects contributing to unquantified transitivity in this NMA and should be consistently reported in future RCTs ${ }^{27,58}$. Three studies had some degree of cross-over between PP and CW arms ${ }^{2,5,54}$ and one study that would otherwise have met the inclusion criteria was excluded due to patients being stratified to either CW or PP in both arms (comparing antecolic and retrocolic routes), with breakdown of data unavailable upon contacting the authors. While many trials focused on comparing a single aspect of $\mathrm{PD}$, adequate consistency between trial arms pertaining to other aspects of PD allowed NMA to be performed. This highlights a strength of this methodology to perform comparisons not feasible in a trial setting

The mechanisms of DGE after PD are multifactorial and remain unresolved. Gastric accommodation and the resultant common cavity pressure gradient between stomach and small bowel influence liquid phase emptying, while antral contractions are more important in the emptying of solids ${ }^{59}$. Myenteric factors (circular smooth muscle and interstitial cells of Cajal), vagal innervation, antropyloric coordination and hormonal influences all play contributory roles ${ }^{60,61}$. The combination of partial vagotomy, distal gastric resection and gastrojejunostomy also predispose to $\mathrm{DGE}^{62}$. Reduced motilin arising from the duodenal resection may also contribute to retention through suppression of the migrating motor complex ${ }^{63}$.

The distal gastric antrum is electrically and functionally distinct to the rest of the stomach, with a specific bioelectrical activity that coordinates the 'terminal antral contraction', being important for trituration and solid gastric emptying ${ }^{64,65}$. CW, with resection of approximately the distal $4 \mathrm{~cm}$ of the stomach, could therefore result in impaired solid gastric emptying due to loss of the terminal antral contraction ${ }^{64}$. Alternatively, potential denervation of the antroduodenal complex as in PR PD could contribute to retention of a relatively hypomotile distal antral remnant that could be counterproductive for gastric emptying. Some authors have advocated that complete antropyloric resection (as in CW) may be preferable for reducing DGE ${ }^{11,54}$, but this proposed advantage of CW was not evident in three other RCTs ${ }^{45,47,52}$ nor the present results, with the highest-ranked technique associated with reduced DGE being a PR PD technique. Experimental evidence, suggesting that coordination of contractions between antrum and pylorus, and pylorus and duodenum is less important for gastric emptying compared with the depth of antral contractions (important for trituration) and receptive relaxation of the small bowel, lends some support to the advantages of antral preservation regarding $\mathrm{DGE}^{66}$.

The various anatomical differences that result from the resection and reconstruction configurations for PD have been widely examined in relation to their roles in $\mathrm{DGE}^{16,67}$. Some hypothesize excess torsion or poor angulation of the gastroenteric anastomosis may be mechanical factors that contribute to $\mathrm{DGE}^{68}$.
Mechanical obstruction caused by a tight transmesocolic window ${ }^{44}$ might be reflected in the poor performance of approaches that use retrocolic gastroenteric anastomosis for DGE in the present study. If bile reflux into the stomach contributes to DGE through mucosal irritation ${ }^{69}$, then this could explain the advantage of Braun enteroenterostomy which allows for bile diversion, provides two paths for gastric contents and stabilizes the gastro-/ duodeno-enteric anastomosis reducing torsion and risk of gastroenteric obstruction. Braun enteroenterostomy may additionally reduce pressure on the small bowel side of the anastomosis, considered important for gastric emptying ${ }^{66}$. A recent report also raised the possibility of aberrant conduction pathways arising from interstitial cells of Cajal regrowth in small-bowel to stomach anastomosis ${ }^{70}$, potentially contributing to DGE regardless of choice of anastomotic configuration, but further clinical data are needed.

A key consideration in mitigating DGE after PD is the incidence of other postoperative complications. POPF, in particular, is a major risk factor for DGE after $\mathrm{PD}^{8,55,71-74}$, so an approach to $\mathrm{PD}$ that also minimizes other complications is an important consideration. In this NMA, PP, retrocolic, Billroth II with Braun enteroenterostomy consistently ranked the best in minimizing rates of POPF, intra-abdominal abscesses, bile leak, PPH and intraoperative blood loss, although it is acknowledged that the included trials were powered to detect differences in DGE, not these other complications. This contrasted with the optimal approach for minimizing DGE (PR, antecolic, Billroth II with Braun enteroenterostomy). One potential explanation for the preference of the antecolic route in DGE could be the separation from the pancreas afforded by the transverse colon, protecting against the local inflammatory consequences of POPF on DGE ${ }^{17}$. Severity of complications has also been correlated with $\mathrm{DGE}^{75}$, so future trials should conform to standardized reporting guidelines to allow for comparisons between trial cohorts ${ }^{25,26}$.

The present analysis did not consider variations in technique for the pancreaticoenteric anastomosis. Gastric and enteric resection and anastomotic factors were prioritized as they were considered more likely to impact on DGE. Despite this, based on existing RCT evidence, a pylorus-resecting, antecolic gastrojejunostomy with Braun enteroenterostomy was associated with the lowest rates of DGE after PD.

\section{Funding}

This study was supported by the New Zealand Health Research Council and the Royal Australasian College of Surgeons John Mitchell Crouch Fellowship. They played no role in performing the systematic review.

Disclosure. Prof. Gregory O'Grady is a member of The University of Auckland Spin-out companies: The Insides Company Ltd, FlexiMap Ltd and Alimetry Ltd.

\section{Supplementary material}

Supplementary material is available at BJS Open online

\section{References}

1. Welsch T, Borm M, Degrate L, Hinz U, Büchler MW, Wente MN et al. Evaluation of the International Study Group of Pancreatic Surgery definition of delayed gastric emptying after 
pancreatoduodenectomy in a high-volume centre. $\mathrm{Br} J$ Surg 2010;97:1043-1050

2. Eshuis WJ, van Eijck CHJ, Gerhards MF, Coene PP, de Hingh IHJT, Karsten TM et al. Antecolic versus retrocolic route of the gastroenteric anastomosis after pancreatoduodenectomy: a randomized controlled trial. Ann Surg 2014;259:45-51

3. Hackert T, Probst P, Knebel P, Doerr-Harim C, Bruckner T, Klaiber $\mathrm{U}$ et al. Pylorus resection does not reduce delayed gastric emptying after partial pancreatoduodenectomy: a blinded randomized controlled trial (PROPP Study, DRKS00004191). Ann Surg 2018;267:1021-1027

4. Busquets J, Martín S, Fabregat J, Secanella L, Pelaez N, Ramos E et al. Randomized trial of two types of gastrojejunostomy after pancreatoduodenectomy and risk of delayed gastric emptying (PAUDA trial). Br J Surg 2019;106:46-54

5. Toyama H, Matsumoto I, Mizumoto T, Fujita H, Tsuchida S, Kanbara Y, Kadowaki Y, et al. Influence of the retrocolic versus antecolic route for alimentary tract reconstruction on delayed gastric emptying after pancreatoduodenectomy: a multicenter, noninferiority randomized controlled trial. Ann. Surg 2020;doi: 10.1097/SLA.0000000000004072

6. Eshuis WJ, de Bree K, Sprangers MAG, Bennink RJ, van Gulik TM, Busch ORC et al. Gastric emptying and quality of life after pancreatoduodenectomy with retrocolic or antecolic gastroenteric anastomosis. BrJ Surg 2015;102:1123-1132

7. Tanaka M. Gastroparesis after a pylorus-preserving pancreatoduodenectomy. Surg Today 2005;35:345-350

8. Ahmad SA, Edwards MJ, Sutton JM, Grewal SS, Hanseman DJ, Maithel SK et al. Factors influencing readmission after pancreaticoduodenectomy: a multi-institutional study of 1302 patients. Ann. Surg 2012;256:529-537

9. Schaefer CJ. Cost and outcome of the Whipple procedure. Ann Surg 1995;222:211-212

10. Gordon TA, Burleyson GP, Tielsch JM, Cameron JL. The effects of regionalization on cost and outcome for one general high-risk surgical procedure. Ann Surg 1995;221:43-49

11. Sun Y-L, Gou JJ, Zhang KM, Li WQ, Ma XX, Zhou L, Zhu RT et al. Complete resection of the gastric antrum decreased incidence and severity of delayed gastric emptying after pancreaticoduodenectomy. Hepatobiliary Pancreat Dis Int 2020; doi: 10.1016/j.hbpd.2020.12.001

12. Huang W, Xiong J-J, Wan M-H, Szatmary P, Bharucha S, Gomatos I et al. Meta-analysis of subtotal stomach-preserving pancreaticoduodenectomy us pylorus preserving pancreaticoduodenectomy. World J Gastroenterol2015;21:6361-6373

13. Klaiber U, Probst P, Strobel O, Michalski CW, Dörr-Harim C, Diener MK et al. Meta-analysis of delayed gastric emptying after pylorus-preserving versus pylorus-resecting pancreatoduodenectomy. Br J Surg 2018;105:339-349

14. Qiu J, Li M, Du C. Antecolic reconstruction is associated with a lower incidence of delayed gastric emptying compared to retrocolic technique after Whipple or pylorus-preserving pancreaticoduodenectomy. Medicine 2019;98:e16663

15. Bell R, Pandanaboyana S, Shah N, Bartlett A, Windsor JA, Smith AM et al. Meta-analysis of antecolic versus retrocolic gastric reconstruction after a pylorus-preserving pancreatoduodenectomy. HPB (Oxford) 2015;17:202-208

16. Kamarajah SK, Bundred JR, Alessandri G, Robinson SM, Wilson $\mathrm{CH}$, French JJ et al. A systematic review and network-metaanalysis of gastro-enteric reconstruction techniques following pancreatoduodenectomy to reduce delayed gastric emptying. World J Surg 2020;44:2314-2322
17. Joliat G-R, Labgaa I, Demartines N, Schäfer M, Allemann P. Effect of antecolic versus retrocolic gastroenteric reconstruction after pancreaticoduodenectomy on delayed gastric emptying: a meta-analysis of six randomized controlled trials. Dig Surg 2016; 33:15-25

18. Deng LH, Xiong JJ, Xia Q. Isolated Roux-en-Y pancreaticojejunostomy versus conventional pancreaticojejunostomy after pancreaticoduodenectomy: a systematic review and metaanalysis. J Evid Based Med 2017;10:37-45

19. Klaiber U, Probst P, Knebel P, Contin P, Diener MK, Büchler MW et al. Meta-analysis of complication rates for single-loop versus dual-loop (Roux-en-Y) with isolated pancreaticojejunostomy reconstruction after pancreaticoduodenectomy. Br J Surg 2015; 102:331-340

20. Xiao Y, Hao X, Yang Q, Li M, Wen J, Jiang C. Effect of Billroth-II versus Roux-en-Y reconstruction for gastrojejunostomy after pancreaticoduodenectomy on delayed gastric emptying: a meta-analysis of randomized controlled trials. J Hepatobiliary Pancreat Sci 2020;doi:10.1002/jhbp.828

21. Ma F, Fan Y, Zhang L, Zhao Z, Nie Y, Chen M et al. Roux-en-Y and Billroth II reconstruction after pancreaticoduodenectomy: a meta-analysis of complications. Biomed Res Int 2020;2020 6131968

22. Schorn S, Demir IE, Vogel T, Schirren R, Reim D, Wilhelm D et al. Mortality and postoperative complications after different types of surgical reconstruction following pancreaticoduodenectomy-a systematic review with meta-analysis. Langenbecks Arch Surg 2019;404:141-157

23. Moher D, Liberati A, Tetzlaff J, Altman DG; PRISMA Group. Preferred reporting items for systematic reviews and meta-analyses: the PRISMA statement. PLoS Med 2009;6:e1000097

24. Hutton B, Salanti G, Caldwell DM, Chaimani A, Schmid CH, Cameron $\mathrm{C}$ et al. The PRISMA extension statement for reporting of systematic reviews incorporating network meta-analyses of health care interventions: checklist and explanations. Ann Intern Med 2015;162:777-784

25. Wente MN, Bassi C, Dervenis C, Fingerhut A, Gouma DJ, Izbicki JR et al. Delayed gastric emptying (DGE) after pancreatic surgery: a suggested definition by the International Study Group of Pancreatic Surgery (ISGPS). Surgery 2007;142:761-768

26. Bassi C, Dervenis C, Butturini G, Fingerhut A, Yeo C, Izbicki J et al.; International Study Group on Pancreatic Fistula Definition. Postoperative pancreatic fistula: an international study group (ISGPF) definition. Surgery 2005;138:8-13

27. Dindo D, Demartines N, Clavien P-A. Classification of surgical complications: a new proposal with evaluation in a cohort of 6336 patients and results of a survey. Ann. Surg 2004;240: 205-213

28. Sterne JAC, Savović J, Page MJ, Elbers RG, Blencowe NS, Boutron I et al. RoB 2: a revised tool for assessing risk of bias in randomised trials. BMJ 2019;366:14898

29. gemtc.drugis.org. Bayesian evidence synthesis. https://gemtc.dru gis.org/\#!/analyses (Date accessed: 5 January 2021).

30. Wan X, Wang W, Liu J, Tong T. Estimating the sample mean and standard deviation from the sample size, median, range and/or interquartile range. BMC Med Res Methodol 2014;14:135

31. Luo D, Wan X, Liu J, Tong T. Optimally estimating the sample mean from the sample size, median, mid-range, and/or midquartile range. Stat Methods Med Res 2018;27:1785-1805

32. Sweeting MJ, Sutton AJ, Lambert PC. What to add to nothing? Use and avoidance of continuity corrections in meta-analysis of sparse data. Stat Med 2004;23:1351-1375 
33. Salanti G, Ades AE, Ioannidis JPA. Graphical methods and numerical summaries for presenting results from multiple-treatment meta-analysis: an overview and tutorial. J Clin Epidemiol 2011;64:163-171

34. van Valkenhoef G, Bujkiewicz S, Efthimiou O, Reid D, Stroomberg C, de Keijser J. gemtc.drugis.org: Bayesian evidence synthesis. https://gemtc.drugis.org/signin.html\#!/analyses 2016

35. Chijiiwa K, Imamura N, Ohuchida J, Hiyoshi M, Nagano M, Otani $\mathrm{K}$ et al. Prospective randomized controlled study of gastric emptying assessed by (13)C-acetate breath test after pylorus-preserving pancreaticoduodenectomy: comparison between antecolic and vertical retrocolic duodenojejunostomy. J Hepatobiliary Pancreat Surg 2009;16:49-55

36. Fujieda H, Yokoyama Y, Hirata A, Usui H, Sakatoku Y, Fukaya M et al. Does Braun anastomosis have an impact on the incidence of delayed gastric emptying and the extent of intragastric bile reflux following pancreatoduodenectomy? - A randomized controlled study. Dig Surg 2017;34:462-468

37. Herrera Cabezón J, Sánchez Acedo P, Tarifa Castilla A, Zazpe Ripa C. Delayed gastric emptying following pancreatoduodenectomy: a Roux-en-Y gastrojejunostomy us Billroth II gastrojejunostomy randomized study. Rev Esp Enferm Dig 2019;111:34-39

38. Hwang HK, Lee SH, Han DH, Choi SH, Kang CM, Lee WJ et al. Impact of Braun anastomosis on reducing delayed gastric emptying following pancreaticoduodenectomy: a prospective, randomized controlled trial. J Hepatobiliary Pancreat Sci 2016;23: 364-372

39. Imamura $\mathrm{N}$, Chijiiwa $\mathrm{K}$, Ohuchida J, Hiyoshi M, Nagano M, Otani $\mathrm{K}$ et al. Prospective randomized clinical trial of a change in gastric emptying and nutritional status after a pylorus-preserving pancreaticoduodenectomy: comparison between an antecolic and a vertical retrocolic duodenojejunostomy. HPB (Oxford) 2014;16:384-394

40. Kakaei F, Beheshtirouy S, Nejatollahi SMR, Rashidi I, Asvadi T, Habibzadeh A et al. Effects of adding Braun jejunojejunostomy to standard Whipple procedure on reduction of afferent loop syndrome - a randomized clinical trial. Can J Surg 2015;58: 383-388

41. Kakaei F, Fakhri MA, Azizi A, Asvadi Kermani T, Tarvirdizade K, Sanei B et al. Effects of antecolic versus retrocolic duodenojejunostomy on delayed gastric emptying after pyloric preserving pancreaticoduodenectomy in patients with periampullary tumors. Asian J Surg 2019;42:963-968

42. Kawai M, Tani M, Hirono S, Miyazawa M, Shimizu A, Uchiyama $\mathrm{K}$ et al. Pylorus ring resection reduces delayed gastric emptying in patients undergoing pancreatoduodenectomy. Ann Surg 2011; 253:495-501

43. Ke S, Ding X-M, Gao J, Zhao A-M, Deng G-y, Ma R-L et al. A prospective, randomized trial of Roux-en-Y reconstruction with isolated pancreatic drainage versus conventional loop reconstruction after pancreaticoduodenectomy. Surgery 2013;153: 743-752

44. Kurahara H, Shinchi H, Maemura K, Mataki Y, Iino S, Sakoda M et al. Delayed gastric emptying after pancreatoduodenectomy. J Surg Res 2011;171:e187-e192

45. Lin PW, Lin YJ. Prospective randomized comparison between pylorus-preserving and standard pancreaticoduodenectomy. $\mathrm{Br} \mathrm{J}$ Surg 1999;86:603-607

46. Matsumoto I, Shinzeki M, Asari S, Goto T, Shirakawa S, Ajiki T et al. A prospective randomized comparison between pylorus- and subtotal stomach-preserving pancreatoduodenectomy on postoperative delayed gastric emptying occurrence and long-term nutritional status. J Surg Oncol 2014;109:690-696
47. Seiler CA, Wagner M, Bachmann T, Redaelli CA, Schmied B, Uhl $W$ et al. Randomized clinical trial of pylorus-preserving duodenopancreatectomy versus classical Whipple resection - long term results. BrJ Surg 2005;92:547-556

48. Shimoda M, Kubota K, Katoh M, Kita J. Effect of Billroth II or Roux-en-Y reconstruction for the gastrojejunostomy on delayed gastric emptying after pancreaticoduodenectomy: a randomized controlled study. Ann Surg 2013;257:938-942

49. Tamandl D, Sahora K, Prucker J, Schmid R, Holst J-J, Miholic J et al. Impact of the reconstruction method on delayed gastric emptying after pylorus-preserving pancreaticoduodenectomy: a prospective randomized study. World J Surg 2014;38:465-475

50. Tani M, Kawai M, Hirono S, Okada K-I, Miyazawa M, Shimizu A et al. Randomized clinical trial of isolated Roux-en-Y versus conventional reconstruction after pancreaticoduodenectomy. Br. J. Surg 2014;101:1084-1091

51. Tani M, Terasawa H, Kawai M, Ina S, Hirono S, Uchiyama K et al. Improvement of delayed gastric emptying in pylorus-preserving pancreaticoduodenectomy: results of a prospective, randomized, controlled trial. Ann Surg 2006;243:316-320.

52. Tran KTC, Smeenk HG, van Eijck CHJ, Kazemier G, Hop WC, Greve JWG et al. Pylorus preserving pancreaticoduodenectomy versus standard Whipple procedure: a prospective, randomized, multicenter analysis of 170 patients with pancreatic and periampullary tumors. Ann Surg 2004;240:738-745

53. Vutukuru VR, Gavini S, Chandrakasan C, Musunuru BR, Settipalli S. Addition of Braun enteroenterostomy to standard reconstruction in pancreaticoduodenectomy: impact on early outcomes. Int Surg J 2017;4:3414

54. Yeo CJ, Cameron JL, Sohn TA, Coleman J, Sauter PK, Hruban RH et al. Pancreaticoduodenectomy with or without extended retroperitoneal lymphadenectomy for periampullary adenocarcinoma: comparison of morbidity and mortality and short-term outcome. Ann Surg 1999;229:613-622

55. Qu H, Sun GR, Zhou SQ, He QS. Clinical risk factors of delayed gastric emptying in patients after pancreaticoduodenectomy: a systematic review and meta-analysis. Eur J Surg Oncol 2013;39: 213-223

56. Wu W, Hong X, Fu L, Liu S, You L, Zhou L et al. The effect of pylorus removal on delayed gastric emptying after pancreaticoduodenectomy: a meta-analysis of 2,599 patients. PLoS One 2014;9: e108380

57. Caldwell DM, Ades AE, Higgins JPT. Simultaneous comparison of multiple treatments: combining direct and indirect evidence. BMJ 2005;331:897-900

58. Martignoni ME, Friess H, Sell F, Ricken L, Shrikhande S, Kulli C et al. Enteral nutrition prolongs delayed gastric emptying in patients after Whipple resection. Am J Surg 2000;180:18-23

59. Indireshkumar K, Brasseur JG, Faas H, Hebbard GS, Kunz P, Dent J et al. Relative contributions of "pressure pump" and "peristaltic pump" to gastric emptying. Am J Physiol Gastrointest Liver Physiol 2000;278:G604-G616

60. Sathar S, Trew ML, OGrady G, Cheng LK. A multiscale tridomain model for simulating bioelectric gastric pacing. IEEE Trans Biomed Eng 2015;62:2685-2692

61. Goyal RK, Guo Y, Mashimo H. Advances in the physiology of gastric emptying. Neurogastroenterol Motil 2019;31:e13546

62. Camilleri M, Parkman HP, Shafi MA, Abell TL, Gerson L; American College of Gastroenterology. Clinical guideline: management of gastroparesis. Am J Gastroenterol 2013;108:18-37

63. Deloose E, Janssen P, Depoortere I, Tack J. The migrating motor complex: control mechanisms and its role in health and disease. Nat Rev Gastroenterol Hepatol 2012;9:271-285 
64. Berry R, Miyagawa T, Paskaranandavadivel N, Du P, Angeli TR, Trew ML et al. Functional physiology of the human terminal antrum defined by high-resolution electrical mapping and computational modeling. Am J Physiol Gastrointest Liver Physiol 2016;311:G895-G902

65. Carlson HC, Code CF, Nelson RA. Motor action of the canine gastroduodenal junction: a cineradiographic, pressure, and electric study. Am J Dig Dis 1966;11:155-172

66. Keinke O, Schemann M, Ehrlein H-J. Mechanical factors regulating gastric emptying of viscous nutrient meals in dogs. Q J Exp Physiol 1984;69:781-795

67. Huang M-Q, Li M, Mao J-Y, Tian B-L. Braun enteroenterostomy reduces delayed gastric emptying: a systematic review and meta-analysis. Int J Surg 2015;23:75-81

68. Barreto SG, Windsor JA. Does the ileal brake contribute to delayed gastric emptying after pancreatoduodenectomy? Dig Dis Sci 2017;62:319-335

69. Vogel SB, Drane WE, Woodward ER. Clinical and radionuclide evaluation of bile diversion by Braun enteroenterostomy: prevention and treatment of alkaline reflux gastritis. An alternative to Roux-en-Y diversion. Ann Surg 1994;219:458-465

70. Wang TH-H, Angeli TR, Beban G, Du P, Bianco F, Gibbons SJ et al. Slow-wave coupling across a gastroduodenal anastomosis as a mechanism for postsurgical gastric dysfunction: evidence for a "gastrointestinal aberrant pathway." Am J Physiol Gastrointest Liver Physiol 2019;317:G141-G146.

71. Kurahara H, Takao S, Shinchi H, Mataki Y, Maemura K, Sakoda $\mathrm{M}$ et al. Subtotal stomach-preserving pancreaticoduodenectomy (SSPPD) prevents postoperative delayed gastric emptying. J Surg Oncol 2010;102:615-619

72. Lermite E, Pessaux P, Brehant O, Teyssedou C, Pelletier I, Etienne $\mathrm{S}$ et al. Risk factors of pancreatic fistula and delayed gastric emptying after pancreaticoduodenectomy with pancreaticogastrostomy. J Am Coll Surg 2007;204: 588-596

73. Ueno T, Tanaka A, Hamanaka Y, Tsurumi M, Suzuki T. A proposal mechanism of early delayed gastric emptying after pylorus preserving pancreatoduodenectomy. Hepatogastroenterology 1995;42:269-274

74. Parmar AD, Sheffield KM, Vargas GM, Pitt HA, Kilbane EM, Hall BL et al. Factors associated with delayed gastric emptying after pancreaticoduodenectomy. HPB 2013;15:763-772

75. El Nakeeb A, Askr W, Mahdy Y, Elgawalby A, El Sorogy M, Abu Zeied $\mathrm{M}$ et al. Delayed gastric emptying after pancreaticoduodenectomy. Risk factors, predictors of severity and outcome. A single center experience of 588 cases. J Gastrointest Surg 2015;19: 1093-1100 